Please quote as: Please quote as: Janson, A. \& Söllner, M. (2017): How TechnologyEnhanced Scaffolding Contributes to Problem-Solving Outcomes in Management Education. In: Academy of Management Annual Meeting (AOM). Atlanta, Georgia, USA. 


\title{
How Technology-Enhanced Scaffolding Contributes to \\ Problem-Solving Outcomes in Management Education
}

\author{
Authors \\ Andreas Janson'1, Matthias Söllner ${ }^{1,2}$ \\ ${ }^{1}$ University of Kassel \\ Information Systems, Research Center for IS Design (ITeG) \\ E-Mail: [andreas.janson, soellner]@uni-kassel.de \\ ${ }^{2}$ University of St. Gallen \\ Institute of Information Management \\ E-Mail: matthias.soellner@ unisg.ch
}

\section{Acknowledgements:}

This research project was partially funded by the German Federal Ministry of Education and Research (BMBF) within the projects StaySmart (02L12A170) and ExTEND (02K14A172), both managed by the Project Management Agency Karlsruhe (PTKA). The authors are responsible for the content of this publication. 


\title{
How Technology-Enhanced Scaffolding Contributes to \\ Problem-Solving Outcomes in Management Education
}

\begin{abstract}
IT support is a key factor for the success of innovative teaching/learning approaches such as flipped classrooms concerned with problem solving. However, IT and the complexity of problem solving may overwhelm the learner and can lead to unsatisfying learning outcomes. Therefore, research suggests the implementation of technology-enhanced scaffolds to guide the learners in their individual problem-solving process to enhance their learning outcomes. To investigate how scaffolding contributes to the learning outcomes in a flipped classroom, we first developed a theoretical model based on adaptive structuration and cognitive load theory. Second, to evaluate the model, we conducted a fully randomized between-subject experiment with 72 students in a flipped classroom for management education focusing on individual problem solving. For this purpose, we accordingly implemented technology-enhanced scaffolds in an LMS, which focused on individual problem-solving activities. Our results show that technology-enhanced scaffolding contributes significantly to the management of cognitive load as well as to learning process satisfaction and problem-solving learning outcomes. In addition, the faithfulness of appropriation and germane load contribute to problem-solving learning outcomes. Therefore, we contribute to both theory and practice by providing a better understanding of technology-enhanced scaffolds and their design to engage problem solving and their respective effects.
\end{abstract}

\section{Keywords:}

Technology-Mediated Learning; Flipped Classroom; Scaffolding; Problem Solving 